\title{
Integrated all-optical SSB modulator using photonic Hilbert transformer with planar Bragg gratings
}

\author{
C. Sima, J. C. Gates, H. L. Rogers, C. Holmes, M. N. Zervas, P. G. R. Smith \\ Optoelectronics Research Centre, University of Southampton, Southampton SO17 1BJ, United Kingdom
}

Photonic Hilbert transformers (PHT) offer potential for a wide range of applications in telecommunication, information processing and signal analysis [1]. They provide operational bandwidths and speeds far beyond current electronic technologies. PHTs have been realized in discrete free space components and recently in fiber using multi-tapped sampled fiber Bragg gratings (FBGs), and single phase-shifted FBGs with appropriate apodisation profile [2]. Recently we have presented preliminary spectra from a fabricated PHT device in a planar waveguide [3]. This device employs a single $\pi$-phase shift and an apodised grating profile. In this work we use the freedom of the planar format to fabricate a single-side-band suppression (SSBS) module combining regular and phase shifted gratings on a single chip.

These devices are fabricated by direct UV grating writing (DGW) technology on a silica-on-silicon platform. This method involves focusing two crossed laser beams (diameter $\sim 6 \mu \mathrm{m}$ ) into the photosensitive core of a planar sample. UV irradiation $(\lambda=244 \mathrm{~nm})$ increases the refractive index in this layer. Precise translation of the sample and modulation of the laser intensity both defines the channel waveguide and simultaneously creates the grating structure. This technique provides the unique ability to insert phase shifts and change the modulation strength of the grating at the micron level. The PHT realized using this technique has an optimized apodised planar Bragg grating with a $\pi$-phase shift in the grating section [4]. An ideal Hilbert transform spectrum exhibits a constant amplitude response and an abrupt $\pi$-phase shift along the wavelength. The experimental data of the current PHT is presented in Figure 1.
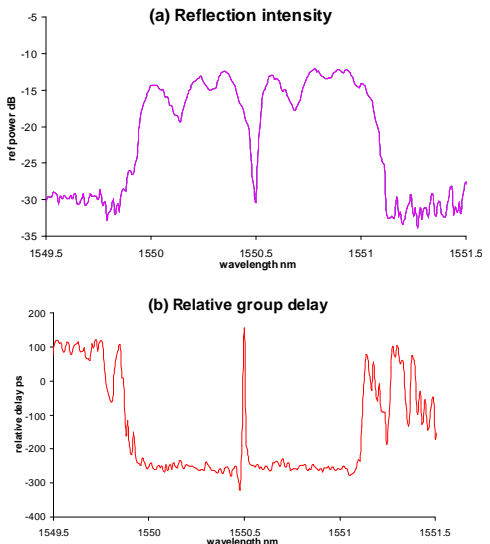

Fig. 1 Reflection response (a) and group delay (b) of the preliminary PHT device of length $15 \mathrm{~mm}$ and designed to operate at $1550.5 \mathrm{~nm}$.

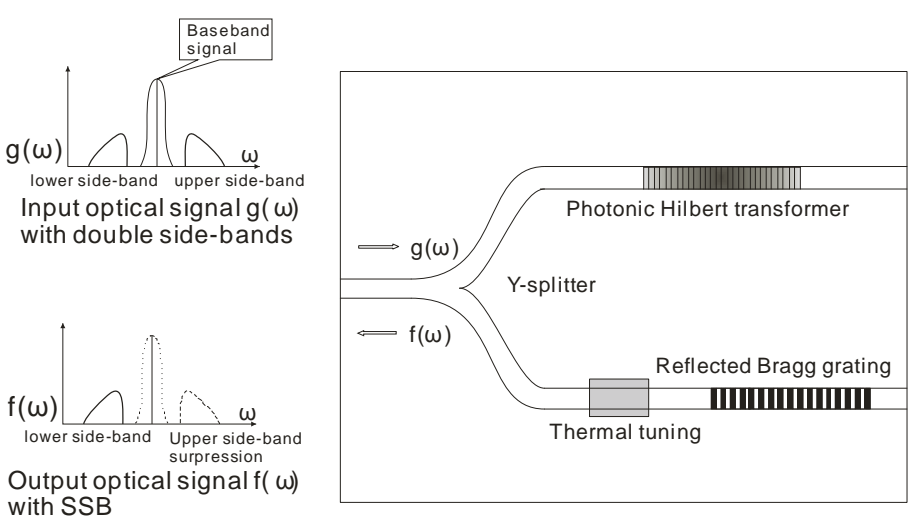

Fig. 2 The proposed structure of integrated device with PHT and gratings in the planar waveguide.

The integrated SSBS module shown in Figure 2 comprises of a Y-splitter, with a PHT and a flat-top band pass reflected grating in each arm. The reflected optical signal from the grating structures undergoes constructive and destructive interference, thereby suppressing one side band and enhancing the other. The relative optical phase of the two signals is tuned by a small thermal element present on the reference arm of the device to allow optimized side band suppression and to select which side band is suppressed.

The fabricated devices feature the integration of couplers, splitters and apodised Bragg gratings within one single chip. We shall present our fabricated devices and specifically their spectral and temporal response functions measured using RF modulated signals.

\section{References}

[1] S. L. Hahn and A. D. Poularikas, the Transforms and Applications Handbook, 2nd ed., (CRC Press LLC, Boca Raton, 2000).

[2] M. Li and J. P. Yao, "Experimental demonstration of a wideband photonic temporal Hilbert transformer based on a single fiber Bragg grating", IEEE Photon. Technol. Lett.22, 1529(2010)

[3] C. Sima, J. C. Gates, B. D. Snow, H. L. Rogers, M. N. Zervas and P. G. R. Smith. "Realisation of photonic Hilbert transformer with a simple planar Bragg grating", Photon 10, Southampton, UK(2010)

[4] M. H. Asghari and J. Azaña, "All-optical Hilbert transformer based on a single phase-shifted fiber Bragg grating: design and analysis", Opt. Lett. 34, 334 (2009). 